\title{
Fast and Accurate Solutions of Extremely Large Scattering Problems Involving Three-Dimensional Canonical and Complicated Objects
}

\author{
Özgür Ergül ${ }^{1,2}$ and Levent Gürel ${ }^{1,2}$ \\ ${ }^{1}$ Department of Electrical and Electronics Engineering \\ ${ }^{2}$ Computational Electromagnetics Research Center (BiLCEM) \\ Bilkent University, TR-06800, Bilkent, Ankara, Turkey \\ ergul@ee.bilkent.edu.tr, lgurel@ee.bilkent.edu.tr
}

\begin{abstract}
We present fast and accurate solutions of extremely large scattering problems involving three-dimensional metallic objects discretized with hundreds of millions of unknowns. Solutions are performed by the multilevel fast multipole algorithm, which is parallelized efficiently via a hierarchical partition strategy. Various examples involving canonical and complicated objects are presented in order to demonstrate the feasibility of accurately solving large-scale problems on relatively inexpensive computing platforms without resorting to approximation techniques.
\end{abstract}

\section{INTRODUCTION}

The multilevel fast multipole algorithm (MLFMA) is a powerful method, which enables the solution of large-scale electromagnetics problems discretized with large numbers of unknowns [1]. This method reduces the complexity of matrix-vector multiplications required by iterative solvers from $\mathcal{O}\left(N^{2}\right)$ to $\mathcal{O}(N \log N)$, where $N$ is the number of unknowns, without deteriorating the accuracy of results. Nevertheless, accurate solutions of real-life problems often require discretizations involving millions of unknowns, which cannot be handled easily with sequential implementations of MLFMA running on a single processor. In order to solve such large problems, it is helpful to increase computational resources by assembling parallel computing platforms and by parallelizing MLFMA.

Among various parallelization schemes for MLFMA, the most popular use distributed-memory architectures by constructing clusters of computers with local memories connected via fast networks [2]-[9]. Unfortunately, parallelization of MLFMA is not trivial, and simple parallelization strategies usually fail to provide efficient solutions. Consequently, there have been many efforts to improve the parallelization of MLFMA by developing novel strategies. Recently, we proposed a hierarchical parallelization strategy, which is based on the optimal partitioning at each level of MLFMA [5],[9]. This strategy offers a higher parallelization efficiency than previous approaches, due to the improved load-balancing and the reduced number of communication events.

In this paper, we present rigorous solutions of extremely large scattering problems involving three-dimensional canon- ical and complicated metallic objects. By employing an efficient parallel implementation of MLFMA, we are able to solve electromagnetics problems discretized with hundreds of millions of unknowns both efficiently and accurately. We present various examples to demonstrate the feasibility of accurately solving large-scale problems on relatively inexpensive computing platforms.

\section{Simulation ENVIRONMENT}

MLFMA and its efficient parallelization via the hierarchical partitioning strategy are extensively discussed in [10],[9]. In this section, we only summarize the major stages of MLFMA, as well as its computational requirements, parallelization, and error sources in the context of developing an efficient and accurate simulation environment.

\section{A. Major Stages of MLFMA}

MLFMA starts with the recursive clustering of the object. A tree structure of $L=\mathcal{O}(\log N)$ levels is constructed by considering nonempty clusters. Before the iterative solution, near-field interactions are calculated in the setup part and stored in memory. Those interactions are between basis and testing functions located in the same cluster or in two touching clusters at the lowest level of the tree structure. In order to calculate far-field interactions on the fly in each matrixvector multiplication, radiation and receiving patterns of basis and testing functions, as well as translation operators for cluster-cluster interactions are also calculated in the setup part. Finally, the right-hand-side vector and the preconditioner to accelerate the iterative solution are prepared before iterations.

During the iterative solution part, matrix-vector multiplications required by the iterative solver are performed efficiently by MLFMA. Far-field interactions are calculated in a groupby-group manner in three main stages, namely, aggregation, translation, and disaggregation, performed on the multilevel tree structure. In the aggregation stage, radiated fields of clusters are calculated from the bottom of the tree structure to the highest level. Then, in the translation stage, radiated fields are translated into incoming fields. Finally, in the disaggregation stage, total incoming fields at cluster centers are calculated 
from the top of the tree structure to the lowest level. At the lowest level, incoming fields are received by testing functions to complete the matrix-vector multiplication.

\section{B. Computational Requirements}

In terms of the processing time, the setup part of MLFMA is dominated by the calculation of near-field interactions. For accurate solutions, those interactions are calculated carefully by employing singularity-extraction techniques [11] [13], adaptive integration methods [14], and Gaussian quadratures [15]. Calculation of radiation and receiving patterns of basis and testing functions, translation operators, and the righthand-side vector requires negligible time compared to the calculation near-field interactions. For all problems presented in this paper, iterative solutions are accelerated with the blockdiagonal preconditioner (BDP), which can also be constructed in negligible time.

In our MLFMA implementation, a majority of memory is used for near-field interactions, radiation and receiving patterns of basis and testing functions, and aggregation/disaggregation arrays required during matrix-vector multiplications. As opposed to common implementations of MLFMA, we calculate and store radiation and receiving patterns of basis and testing functions during the setup of the program, and we use them efficiently during iterations. Calculating the patterns on the fly in each matrix-vector multiplication without storing them would decrease the memory requirement; but the processing time would increase significantly.

\section{Parallelization}

MLFMA can be parallelized efficient by using a hierarchical partitioning strategy, which is based on the simultaneous partitioning of clusters and their fields at all levels [5],[9]. We adjust the partitioning in both directions, i.e., clusters and samples of radiated and incoming fields, appropriately by considering the number of clusters and the number of samples at each level. The hierarchical partitioning strategy provides two important advantages, compared to previous parallelization techniques for MLFMA. First, partitioning both clusters and samples of fields leads to an improved loadbalancing of the workload among processors at each level. Second, communications between processors are reduced, i.e, average package size is enlarged, the number of communication events is reduced, and the communication time is significantly shortened.

\section{Error Sources}

In MLFMA implementations, there are various error sources, which must be controlled carefully for accurate solutions. Basically, the simultaneous discretization (triangulation) of surfaces and integral-equation formulations constitutes the major error source in numerical solutions. Using the RaoWilton-Glisson (RWG) functions [16] for the discretization, triangles should be much smaller than the wavelength. Typically, in order to obtain less than $1 \%$ error, the largest triangle should be smaller than $\lambda / 10$, where $\lambda$ is the wavelength.
TABLE I

SOlutions of EXTREMEly LARGE SCATTERING Problems INVOLVING CANONicAl ANd COMPlicated OBJects Depicted In Fig. 1

\begin{tabular}{|c|c|c|c|c|c|c|}
\hline Object & \multicolumn{6}{|c|}{ Rectangular Box } \\
\hline Size & \multicolumn{6}{|c|}{$875 \lambda$} \\
\hline Number of Unknowns & \multicolumn{6}{|c|}{$174,489,600$} \\
\hline Polarization & \multicolumn{3}{|c|}{$\phi$} & \multicolumn{3}{|c|}{$\theta$} \\
\hline Illumination Angle & $30^{\circ}$ & $60^{\circ}$ & $90^{\circ}$ & $30^{\circ}$ & $60^{\circ}$ & $90^{\circ}$ \\
\hline Number of Iterations & 15 & 17 & 36 & 16 & 36 & 36 \\
\hline Total Time (minutes) & 269 & 291 & 493 & 280 & 493 & 493 \\
\hline$\overline{\text { Object }}$ & \multicolumn{6}{|c|}{ NASA Almond } \\
\hline Size & \multicolumn{6}{|c|}{$915 \lambda$} \\
\hline Number of Unknowns & \multicolumn{6}{|c|}{$203,476,224$} \\
\hline Polarization & \multicolumn{3}{|c|}{$\phi$} & \multicolumn{3}{|c|}{$\theta$} \\
\hline Illumination Angle & $30^{\circ}$ & $60^{\circ}$ & $90^{\circ}$ & $30^{\circ}$ & $60^{\circ}$ & $90^{\circ}$ \\
\hline Number of Iterations & 20 & 17 & 17 & 27 & 24 & 28 \\
\hline Total Time (minutes) & 442 & 515 & 515 & 676 & 628 & 693 \\
\hline Object & \multicolumn{6}{|c|}{ Flamme } \\
\hline Size & \multicolumn{6}{|c|}{$880 \lambda$} \\
\hline Number of Unknowns & \multicolumn{6}{|c|}{$204,664,320$} \\
\hline Polarization & \multicolumn{3}{|c|}{$\phi$} & \multicolumn{3}{|c|}{$\theta$} \\
\hline Illumination Angle & $30^{\circ}$ & $60^{\circ}$ & $90^{\circ}$ & $30^{\circ}$ & $60^{\circ}$ & $90^{\circ}$ \\
\hline Number of Iterations & 37 & 42 & 88 & 46 & 80 & 154 \\
\hline Total Time (minutes) & 869 & 951 & 1753 & 1019 & 1611 & 2922 \\
\hline
\end{tabular}

Accurate solutions also require accurate computations of matrix elements. Using adaptive integration methods [14], we calculate near-field interactions with a maximum of $1 \%$ error. Far-field interactions are also calculated with a maximum of $1 \%$ error using the excess bandwidth formula to determine truncation numbers and sampling rates for radiated and incoming fields [17]. Interpolation and anterpolation operations constitute another error source in MLFMA. We use various techniques in order to suppress those additional errors without deteriorating the efficiency of the implementation [18],[19]. Finally, the relative residual error for the iterative convergence is typically set to $10^{-3}$ in all solutions.

\section{RESULTS}

In this paper, we present solutions of scattering problems involving three metallic objects depicted in Fig. 1, namely, a $1 \mathrm{~m} \times 3.5 \mathrm{~m} \times 0.1 \mathrm{~m}$ rectangular box, the NASA Almond [20] of length $25.23 \mathrm{~cm}$, and the complicated stealth airborne target Flamme [21] with a maximum dimension of $0.6 \mathrm{~m}$. All three objects involve closed conducting surfaces, which can be formulated with the combined-field integral equation (CFIE) [22].

The rectangular box is investigated at $75 \mathrm{GHz}$. At this frequency, the size of the box corresponds to $875 \lambda$, and its discretization with the RWG functions on $\lambda / 10$ triangles leads to matrix equations involving $174,489,600$ unknowns. The NASA Almond is discretized with 203,476,224 unknowns and investigated at $1.1 \mathrm{THz}$. The maximum dimension of the NASA Almond corresponds to $915 \lambda$ at this frequency. Finally, the Flamme is discretized with 204,664,320 unknowns and investigated at $440 \mathrm{GHz}$. The scaled size of the Flamme is $0.6 \mathrm{~m}$, which corresponds to $880 \lambda$ at this frequency.

The rectangular box is illuminated by three plane waves propagating on the $x-z$ plane at $30^{\circ}, 60^{\circ}, 90^{\circ}$ angles from 

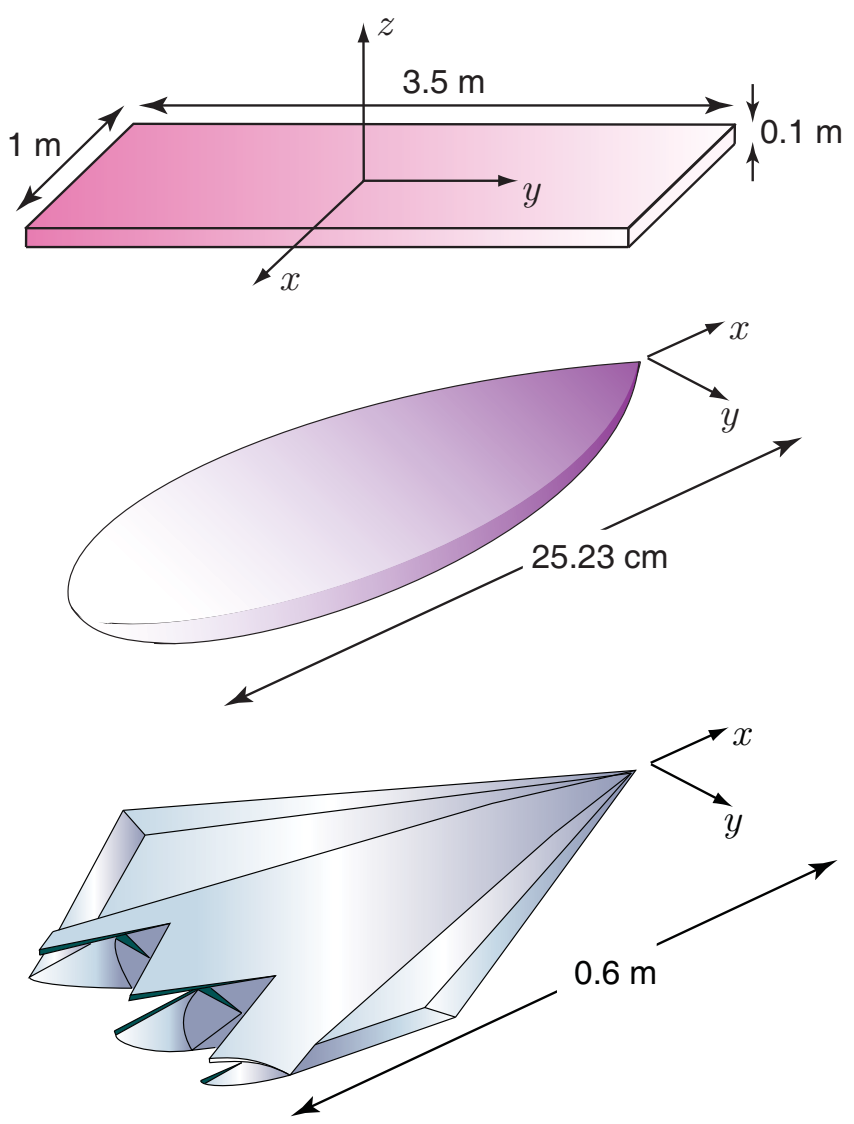

Fig. 1. Large metallic objects, whose scattering problems are solved by the parallel MLFMA implementation.

the $z$ axis with the electric field polarized in the $\theta$ direction. The NASA Almond and the Flamme are illuminated by three plane waves propagating on the $x-y$ plane at $30^{\circ}, 60^{\circ}$, and $90^{\circ}$ angles from the $x$ axis (from the nose) with the electric field polarized in the $\phi$ direction. Solutions are performed on a cluster of Intel Xeon Dunnington processors with $2.40 \mathrm{GHz}$ clock rate. The cluster consists of 16 computing nodes, and each node has 48 GB of memory and multiple processors. For all solutions, we employ four cores per node (a total of 64 cores). Iterative solutions are performed by using the biconjugate-gradient-stabilized algorithm [23] accelerated via BDP.

Table I lists the number of iterations and the total processing time for each solution. For the Flamme, the number of iterations significantly increases with the increasing illumination angle, due to the resonant characteristics of the cavity at the back of the target. This is not observed for the NASA Almond, which has a convex surface with a more regular shape. In the case of the rectangular box, solutions require more iterations for oblique incidences. Using a total of $768 \mathrm{~GB}$ memory of the Dunnington cluster, rectangular-box problems are solved in 4.5-8.5 hours, NASA-Almond problems are solved in 7.511.5 hours, and Flamme problems are solved in 14.5-49.0 hours.
Fig. 2 depicts the bistatic radar cross section RCS (dBms) of the rectangular box on the $x-z$ plane as a function of the bistatic angle $\theta$. For $30^{\circ}$ and $60^{\circ}$ illuminations, RCS values make peaks at two reflection directions, in addition to the forward-scattering direction. For the $90^{\circ}$ illumination, a reflection direction coincides with the back-scattering direction. We note that the cross-polar RCS is significantly lower than the co-polar RCS of the rectangular box for all illuminations.

Fig. 3 depicts the bistatic RCS (dBms) of the NASA Almond on the $x-y$ plane as a function of the bistatic angle $\phi$. We observe that the NASA Almond has a stealth ability with very low back-scattered RCS compared to the forwardscattered RCS. Specifically, for the $30^{\circ}$ illumination, the backscattered RCS is $95 \mathrm{~dB}$ lower than the forward-scattered RCS. This large difference decreases to about $77 \mathrm{~dB}$ for $60^{\circ}$ and $90^{\circ}$ illuminations. We also note that, depending on the illumination, RCS of the NASA Almond is relatively high in a range of bistatic directions, i.e., $90^{\circ}-210^{\circ}, 60^{\circ}-240^{\circ}$, and $30^{\circ}-270^{\circ}$ for $30^{\circ}, 60^{\circ}$ and $90^{\circ}$ illuminations, respectively.

Finally, Fig. 4 depicts the bistatic RCS (dBms) of the stealth airborne target Flamme on the $x-y$ plane as a function of the bistatic angle $\phi$. For $30^{\circ}$ and $60^{\circ}$ illuminations, the backscattered RCS of the Flamme is very low, i.e., it is $90 \mathrm{~dB}$ and $105 \mathrm{~dB}$ lower than the forward-scattered RCS, respectively. For the $90^{\circ}$ illumination, however, the difference between RCS values in the back-scattering and forward-scattering directions decreases to $56 \mathrm{~dB}$. As opposed to the RCS of the NASA Almond, the Flamme RCS exhibits several peaks in various directions depending on the illumination. In addition, the crosspolar RCS of the Flamme is quite significant and comparable to its co-polar RCS for all illuminations.

\section{CONCLUSION}

This paper presents rigorous solutions of scattering problems involving metallic objects discretized with hundreds of millions of unknowns using a parallel implementation of MLFMA. Using a hierarchical partitioning strategy, those large-scale problems can be solved easily on relatively inexpensive computing platforms without resorting to approximation techniques.

\section{ACKNOWLEDGMENT}

This work was supported by the Turkish Academy of Sciences in the framework of the Young Scientist Award Program (LG/TUBA-GEBIP/2002-1-12), by the Scientific and Technical Research Council of Turkey (TUBITAK) under Research Grants 105E172 and 107E136, and by contracts from ASELSAN and SSM.

\section{REFERENCES}

[1] J. Song, C.-C. Lu, and W. C. Chew, "Multilevel fast multipole algorithm for electromagnetic scattering by large complex objects," IEEE Trans. Antennas Propag., vol. 45, no. 10, pp. 1488-1493, Oct. 1997.

[2] S. Velamparambil, W. C. Chew, and J. Song, "10 million unknowns: Is it that big?," IEEE Antennas Propag. Mag., vol. 45, no. 2, pp. 43-58, Apr. 2003.

[3] S. Velamparambil and W. C. Chew, "Analysis and performance of a distributed memory multilevel fast multipole algorithm," IEEE Trans. Antennas Propag., vol. 53, no. 8, pp. 2719-2727, Aug. 2005. 
[4] L. Gürel and Ö. Ergül, "Fast and accurate solutions of integral-equation formulations discretised with tens of millions of unknowns," Electron. Lett., vol. 43, no. 9, pp. 499-500, Apr. 2007.

[5] Ö. Ergül and L. Gürel, "Hierarchical parallelisation strategy for multilevel fast multipole algorithm in computational electromagnetics," Electron. Lett., vol. 44, no. 1, pp. 3-5, Jan. 2008.

[6] Ö. Ergül and L. Gürel, "Efficient parallelization of the multilevel fast multipole algorithm for the solution of large-scale scattering problems," IEEE Trans. Antennas Propag., vol. 56, no. 8, pp. 2335-2345, Aug. 2008.

[7] J. Fostier and F. Olyslager, "An asynchronous parallel MLFMA for scattering at multiple dielectric objects," IEEE Trans. Antennas Propag., vol. 56, no. 8, pp. 2346-2355, Aug. 2008.

[8] X.-M. Pan and X.-Q. Sheng, "A sophisticated parallel MLFMA for scattering by extremely large targets," IEEE Antennas Propag. Mag., vol. 50, no. 3, pp. 129-138, Jun. 2008 .

[9] Ö. Ergül and L. Gürel, "A hierarchical partitioning strategy for an efficient parallelization of the multilevel fast multipole algorithm," IEEE Trans. Antennas Propag., vol. 57, no. 6, pp. 1740-1750, Jun. 2009.

[10] W. C. Chew, J.-M. Jin, E. Michielssen, and J. Song, Fast and Efficient Algorithms in Computational Electromagnetics. Boston, MA: Artech House, 2001.

[11] R. D. Graglia, "On the numerical integration of the linear shape functions times the 3-D Green's function or its gradient on a plane triangle," IEEE Trans. Antennas Propag., vol. 41, no. 10, pp. 1448-1455, Oct. 1993.

[12] R. E. Hodges and Y. Rahmat-Samii, "The evaluation of MFIE integrals with the use of vector triangle basis functions," Microw. Opt. Technol. Lett., vol. 14, no. 1, pp. 9-14, Jan. 1997.

[13] L. Gürel and Ö. Ergül, "Singularity of the magnetic-field integral equation and its extraction," IEEE Antennas Wireless Propag. Lett., vol. 4, pp. 229-232, 2005.

[14] Ö. S. Ergül, Fast multipole method for the solution of electromagnetic scattering problems, M. S. Thesis, Bilkent University, Ankara, Turkey, Jun. 2003.

[15] D. A. Dunavant, "High degree efficient symmetrical Gaussian quadrature rules for the triangle," Int. J. Numer. Meth. Eng., vol. 21, pp. 1129-1148, 1985.

[16] S. M. Rao, D. R. Wilton, and A. W. Glisson, "Electromagnetic scattering by surfaces of arbitrary shape," IEEE Trans. Antennas Propag., vol. 30, no. 3, pp. 409-418, May 1982

[17] S. Koc, J. M. Song, and W. C. Chew, "Error analysis for the numerical evaluation of the diagonal forms of the scalar spherical addition theorem," SIAM J. Numer. Anal., vol. 36, no. 3, pp. 906-921, 1999.

[18] Ö. Ergül and L. Gürel, "Optimal interpolation of translation operator in multilevel fast multipole algorithm," IEEE Trans. Antennas Propag. vol. 54, no. 12 , pp. 3822-3826, Dec. 2006.

[19] Ö. Ergül and L. Gürel, "Enhancing the accuracy of the interpolations and anterpolations in MLFMA," IEEE Antennas Wireless Propagat. Lett., vol. 5, pp. 467-470, 2006.

[20] A. K. Dominek, M. C. Gilreath, and R. M. Wood, "Almond test body," United States Patent, no. 4809003, 1989.

[21] L. Gürel, H. Bağcı, J. C. Castelli, A. Cheraly, and F. Tardivel, "Validation through comparison: measurement and calculation of the bistatic radar cross section (BRCS) of a stealth target," Radio Sci., vol. 38, no. 3, Jun. 2003.

[22] J. R. Mautz and R. F. Harrington, "H-field, E-field, and combined field solutions for conducting bodies of revolution," $A E \ddot{U}$, vol. 32, no. 4, pp. 157-164, Apr. 1978.

[23] H. A. van der Vorst, "Bi-CGSTAB: A fast and smoothly converging variant of Bi-CG for the solution of nonsymmetric linear systems," SIAM J. Sci. Stat. Comput., vol. 13, no. 2, pp. 631-644, Mar. 1992.
Rectangular Box $(875 \lambda)$
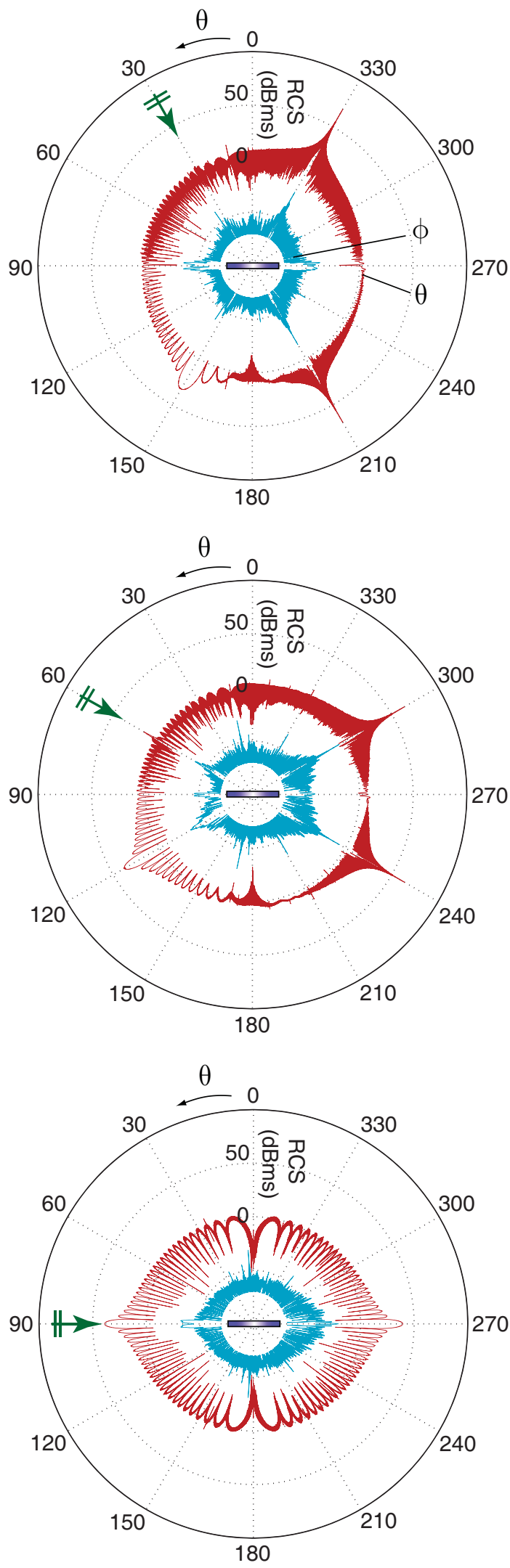

Fig. 2. Bistatic RCS (dBms) of the rectangular box in Fig. 1 at $75 \mathrm{GHz}$ RCS values less than $-70 \mathrm{dBms}$ are omitted. 

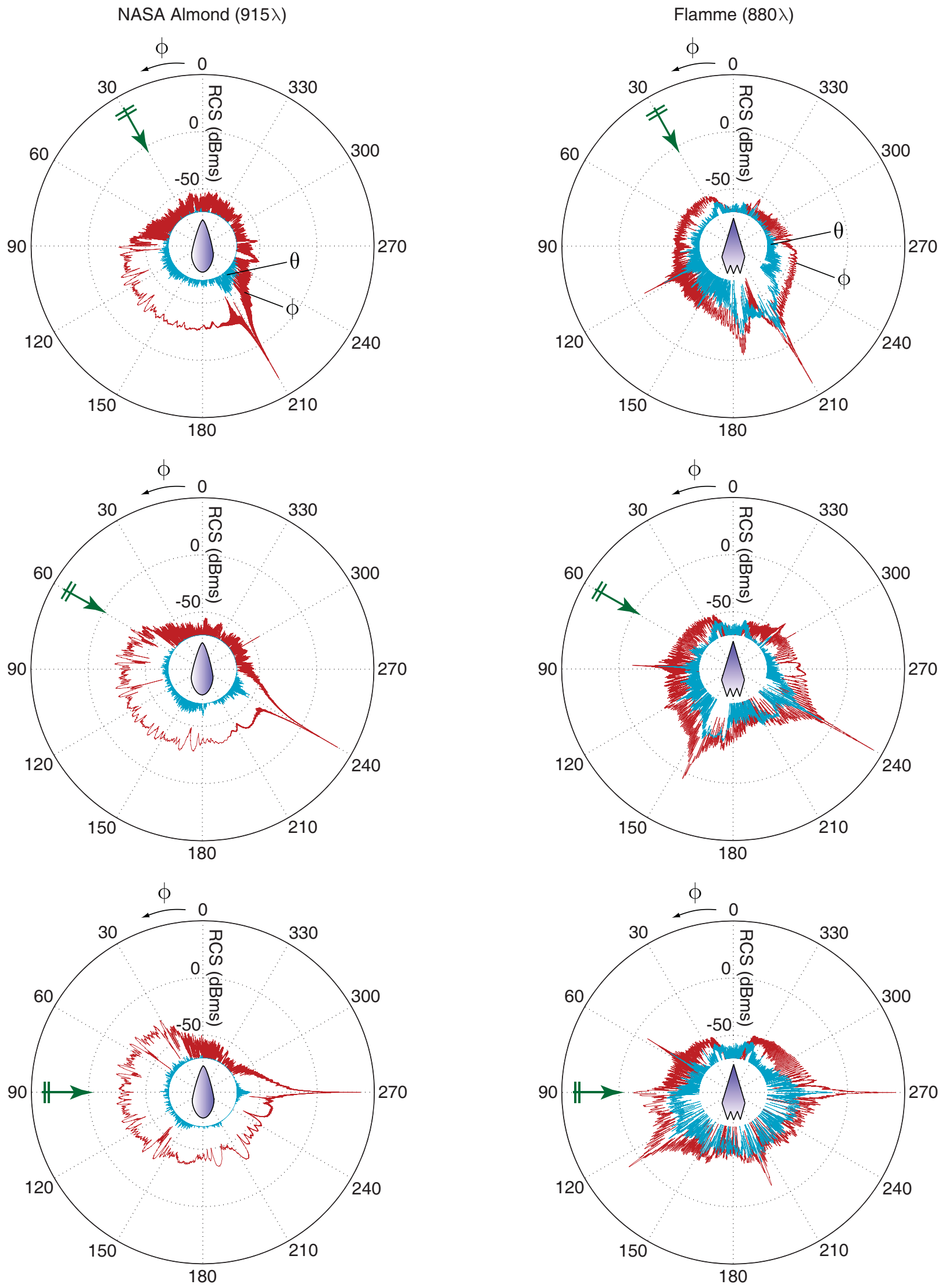

Fig. 3. Bistatic RCS (dBms) of the NASA Almond in Fig. 1 at $1.1 \mathrm{THz}$ RCS values less than $-70 \mathrm{dBms}$ are omitted.

Fig. 4. Bistatic RCS (dBms) of the Flamme in Fig. 1 at $440 \mathrm{GHz}$. RCS values less than $-70 \mathrm{dBms}$ are omitted. 